\title{
Association between Exercise and Metabolic Syndrome in Koreans ( $\mathrm{J}$ Obes Metab Syndr 2018;27:117-24)
}

\author{
Ye An Kim* \\ Department of Internal Medicine, Veterans Health Service Medical Center, Seoul, Korea
}

\author{
Received November 6, 2018 \\ Reviewed November 18, 2018 \\ Accepted December 1, 2018 \\ ${ }^{*}$ Corresponding author \\ Ye An Kim \\ https://orcid.org/0000-0001-8986-2632 \\ Department of Internal Medicine, \\ Veterans Health Service Medical Center, \\ 53 Jinhwangdo-ro 61-gil, Gangdong-gu, \\ Seoul 05368, Korea \\ Tel: +82-2-2225-4691 \\ Fax: +82-2-2225-1433 \\ E-mail: yeanin@gmail.com
}

Physical activity is beneficial for the prevention and treatment of metabolic syndrome. However, when physicians recommend exercise to their patients, there are several factors that need to be considered, such as the type, frequency, and duration of physical activity. In addition, metabolic syndrome is a cluster of conditions, and the effect of exercise may be different between blood glucose or lipid conditions, blood pressure, and abdominal obesity. We have previously investigated the association between physical activity and metabolic syndrome.

Based on the data from the Korea National Health and Nutrition Examination Survey (KNHANES 2007-2009), we showed that the prevalence of metabolic syndrome was significantly lower in the moderate to vigorous exercise group than that in the walking only group and no exercise group. For the metabolic components, the moderate to vigorous exercise group showed a lower risk of abdominal obesity and dyslipidemia. The risk of fasting hyperglycemia tended to be lower in both the intense exercise group and the walking only groups than in the no exercise group, but this was not the case for high blood pressure.

As a reader mentioned, there was another study showing the beneficial effects of exercise on the prevalence of metabolic syndrome using the KNHANES (1999-2012). ${ }^{2}$ The study calculated the prevalence of metabolic syndrome and the mean values of metabolic components according to a frequency of 0 to 7 per week for each exercise type. This study design reflects the original questionnaire, the International Physical Activity Questionnaire short form (IPAQ-SF) with two questions (number of days of flexibility and muscle power exercise per week). In contrast, our study was based on three groups defined by exercise intensity, regardless of frequency. In previous studies on the validity of IPAQ-SF in Koreans, exercise volume tended to be inflated in the intense physical activity group, resulting in a difference between energy expenditure (EE) calculated by the IPAQ-SF and actual EE measured by an accelerometer. ${ }^{3,4}$ The reliability on the time spent walking was low, which may be due to daily variation. ${ }^{4} \mathrm{~A}$ systematic review revealed that the validity of the IPAQ-SF was low, while some acceptable correlations were shown with vigorous activity or walking. ${ }^{5}$ Since the frequency and duration of moderate exercise are strongly correlated with those of vigorous exercise, we combined the respondents who answered that they performed moderate exercise and vigorous exercise. It was also considered that the questionnaire about moderate exercise and vigorous exercise included the same exercise items, such as swimming and tennis. Because the frequency and duration of each physical activity intensity varied and overlapped, they were 
not considered in the definition of the exercise group. In fact, there was no trend in the prevalence of metabolic syndrome according to the frequency and duration of each category of physical activity intensity. Although the prevalence of metabolic syndrome was low among the participants who performed moderate exercise for 5 days per week and vigorous exercise for 6 days per week, it was rather high in those who performed moderate or vigorous exercise for 7 days per week. Regardless of exercise intensity, time spent on exercise at any one time was about 1 hour on average; the total time per week was strongly correlated with the days of exercise, and it was not associated with the prevalence of metabolic syndrome. ${ }^{1}$

As the reader also noted, the effect of exercise on metabolic syndrome can be influenced by several other factors, such as body composition, cardiorespiratory fitness, and being post-menopause. Low muscle mass or abdominal obesity has a negative influence on physical performance. ${ }^{6,7}$ A recent meta-analysis demonstrated not only a preventive effect of physical activity on sarcopenia but also a significant association between sarcopenia and physical activity. ${ }^{8}$ Insulin-like growth factor-1, adipokines, and sex hormones seem to be involved in the mechanisms underlying the relationship between body composition and physical activity., ${ }^{9}$ We performed multiple logistic regressions for the risk of metabolic syndrome and its components in three-stage models with adjustment for age, sex, body mass index, total energy intake, smoking, alcohol intake, and household income. The competence of an exercise participant affects their exercise performance, and the effects of exercise on the body are integrative and multidirectional. It would be interesting to further consider the dynamic factors related to exercise performance and the effect of exercise in association with body composition.

As mentioned at the end of our manuscript, we agree that several studies need to be conducted to establish the best exercise recommendations. This study also started to obtain clues about exercise prescription for patients with metabolic disorders. Although it was a cross-sectional study and the causal relationship was difficult to be determined, many efforts have been made to establish a statistical design that best reflects the real world. Through the reader's insightful perspectives in the letter, we have been able to explain the background of this study design and broaden our views on the association between physical activity and metabolic syndrome. We sincerely appreciate the letter and the opportunity to respond to it for the Journal of Obesity \& Metabolic Syndrome.

\section{CONFLICTS OF INTEREST}

The author declares no conflict of interest.

\section{REFERENCES}

1. Lee SH, Lee Y, Seo JH, Kim YA. Association between exercise and metabolic syndrome in Koreans. J Obes Metab Syndr 2018; 27:117-24.

2. Lee J, Kim Y, Jeon JY. Association between physical activity and the prevalence of metabolic syndrome: from the Korean National Health and Nutrition Examination Survey, 1999-2012. Springerplus 2016;5:1870.

3. Oh JY, Yang YJ, Kim BS, Kang JH. Validity and reliability of Korean version of International Physical Activity Questionnaire (IPAQ) short form. J Korean Acad Fam Med 2007;28:532-41.

4. Chun MY. Validity and reliability of Korean version of international physical activity questionnaire short form in the elderly. Korean J Fam Med 2012;33:144-51.

5. Lee PH, Macfarlane DJ, Lam TH, Stewart SM. Validity of the International Physical Activity Questionnaire Short Form (IPAQ-SF): a systematic review. Int J Behav Nutr Phys Act 2011;8:115.

6. Tanimoto Y, Watanabe M, Sun W, Sugiura Y, Tsuda Y, Kimura $\mathrm{M}$, et al. Association between sarcopenia and higher-level functional capacity in daily living in community-dwelling elderly subjects in Japan. Arch Gerontol Geriatr 2012;55:e9-13.

7. Aleksandrova K, Jenab M, Leitzmann M, Bueno-de-Mesquita B, Kaaks R, Trichopoulou A, et al. Physical activity, mediating factors and risk of colon cancer: insights into adiposity and circulating biomarkers from the EPIC cohort. Int J Epidemiol 2017;46:1823-35.

8. Steffl M, Bohannon RW, Sontakova L, Tufano JJ, Shiells K, Holmerova I. Relationship between sarcopenia and physical activity in older people: a systematic review and meta-analysis. Clin Interv Aging 2017;12:835-45.

9. Spartano NL, Stevenson MD, Xanthakis V, Larson MG, An- 
dersson C, Murabito JM, et al. Associations of objective physical activity with insulin sensitivity and circulating adipokine profile: the Framingham Heart Study. Clin Obes 2017;7:59-69. 10. Timmons BW, Hamadeh MJ, Devries MC, Tarnopolsky MA.
Influence of gender, menstrual phase, and oral contraceptive use on immunological changes in response to prolonged cycling. J Appl Physiol (1985) 2005;99:979-85. 